\title{
Stigma of Mental Illness: Social Distancing Attitudes among Registered Nurses in Australia
}

\author{
Tan Kan Kuㅜ, Michael Ha ${ }^{2}$ \\ ${ }^{1}$ Victoria University Institute of Technology (VUIT), St. Albans Campus, McKechine Street, St. Albans P. O. Box \\ 14428, Melbourne, Victoria 8001, Australia \\ ${ }^{2}$ Financial Mathematics Programme, Xi'an Jiaotong-Liverpool University, Suzhou, China \\ Email: tankanku@gmail.com, Tan.Ku@vu.edu.au, Michael.Ha@xjtlu.edu.cn
}

Received 16 November 2015; accepted 11 December 2015; published 18 December 2015

\begin{abstract}
Background: Stigma of mental illness is often examined in social psychology and psychiatric rehabilitation using attitude studies. Participants of these studies are among health professionals and general public members. A common measure of stigma is using validated scale which measures the opinion on mental illness. Method: A cross-sectional survey was presented to 208 registered nurses. Principal component analyses (with oblique rotation) were used to identify underlying dimensionality in the correlations of items for social distancing. Subscale score variations were analysed across nurse type and ethnicity to examine the discriminant validity of the subscale. Results: Principal component analysis (PCA) revealed one dimension accounting for $43.5 \%$ of the variations within items for social distancing. Developed as scale, termed Stigma towards Psychiatric Patients (STPP), reliability analysis indicated high internal consistency with respective alpha coefficient of 0.8. Chinese general nurses scored highest on social distancing than the other three groups: Chinese psychiatric nurses, Anglo general and Anglo psychiatric nurses. Conclusion: Psychometric evaluation of the Stigma Scale (STPP) suggests it is a reliable instrument for measuring social distancing attitudes towards mental illness. The effect of ethnicity on stigmatising attitudes is not entirely accounted for by exposure to people with mental illness.
\end{abstract}

\section{Keywords}

Stigma, Mental Illness, Social Distancing

\section{Introduction}

The word "stigma" has its origin in Greek and is defined in the dictionary as a mark of disgrace or dishonour; a reproach or slur (New Webster's Dictionary and Thesaurus and Medical Dictionary, 1992) [1]. An illustration of the word "stigma" is its application to the wounds (stigmata in pleural) inflicted on the Lord Jesus when He was crucified. The wounded sores signify that the bearer is a criminal, a slave, a betrayer or a notorious person. "Stigma" is used often in relation to attitudes of shame, guilt, disgrace or disapproval, and fear of identification 
with self and others (Goffman, 1970) [2]. Stigma is a word that stirs up negative emotion and reaction. Stigmatised individuals are likely to be disliked, rejected and discriminated against.

Stigma of mental illness is associated with people who are often perceived by the general public as untrustworthy and incompetent (Angermeyer \& Schulze, 2001) [3] and in severe cases, the stigmatised person is "locked away" and hidden from society (Rabkin, 1974) [4]. A person with mental illness is regarded as dangerous, unpredictable and this in part "justifies" their discrimination by society. The term also implies inner perception of inferiority regarding the self especially after hospitalization and labeling (Link et al., 1991) [5] and leads the individual to behave in such a way that causes others to respond negatively to such self-perception and perpetuate feelings of exclusion and inadequacy. The degree of stigma is particularly strong when the mentally ill person exhibits unusual or extraordinary behaviours, such as episodes of psychosis in Chinese society (Lin, 1983) [6].

\section{Methods}

\subsection{Subjects}

Two hundred and eight nurses (208) participated in this study. They were selected for the purpose of a larger study on the influence of cultural values in nursing practice within psychiatric and general settings. Consequently, the sample was comprised of 49 Chinese-Australian and 83 Anglo-Australian psychiatric nurses, and 35 Chinese-Australian and 41 Anglo-Australian general nurses. One hundred and forty eight (148) were females and 60 were males. The mean age of the sample was 44.8 years (s.d. =9.6), ranging from 21 to 65 years of age. For the overall sample the mean number of years working in general setting was 11.2 (s.d. $=12.0$ ) and in psychiatric setting was 9.8 (s.d. $=11.0$ ) respectively. Table 1 shows the sample demographics.

With regards to whether or not the nurses had received psychiatric training, the results indicated that a large number (over 90\%) of psychiatric nurses in the sample had psychiatric training. As indicated in Table 1, a smaller number of general nurses than psychiatric nurses, and a smaller number of Chinese General nurses than Anglo nurses, had psychiatric training. This difference was statistically significant. Though the majority of nurses underwent nursing training through a course of study ranging between $80 \%$ and $100 \%$, there were no significant differences in relation to training course, in-service training or other forms of experience when the sources of training were compared.

\subsection{Item Development of General Stigma Scale (STPP)}

A reduced version (consisting of 10 questions) of the Opinion on Mental Illness (OMI) Scale developed by Cohen \& Struening (1962) [7] was used to measure social distancing and alienation of those with mental illness,

Table 1. Characteristics of the sample $(\mathrm{N}=208)$.

\begin{tabular}{|c|c|c|c|c|c|}
\hline & $\begin{array}{l}\text { Chinese } \\
\text { Psychiatry } \\
(n=49)\end{array}$ & $\begin{array}{c}\text { Chinese } \\
\text { General } \\
(n=35)\end{array}$ & $\begin{array}{l}\text { Anglo } \\
\text { Psychiatry } \\
(\mathrm{n}=83)\end{array}$ & $\begin{array}{l}\text { Anglo } \\
\text { General } \\
(n=41)\end{array}$ & $\chi^{2 / \mathrm{F}}$ value \\
\hline Age ${ }^{*}($ mean, s.d. $)$ & $50.3(5.8)$ & $47.9(6.0)$ & $43.1(9.6)$ & $39.5(11.6)$ & $13.2 * * * \mathrm{~F}(1,197)$ \\
\hline Sex (male/female) & e) $25 / 24$ & $1 / 34$ & $31 / 52$ & $3 / 38$ & $35.4 * * *$ \\
\hline Psy training (\%) & 95.9 & 28.6 & 90.4 & 61.0 & $67.3 * * *$ \\
\hline Course & 80.9 & 90.0 & 84.0 & 100 & $5.5 \mathrm{~ns}$ \\
\hline In-service & 6.4 & 0 & 12.0 & 0 & $5.0 \mathrm{~ns}$ \\
\hline Others & 12.8 & 10.0 & 6.7 & 0 & $3.9 \mathrm{~ns}$ \\
\hline \multicolumn{5}{|c|}{ Years in Mental Health Nursing (mean, s.d.) } & $\mathrm{F}(1,200)$ \\
\hline & $19.5(10.4)$ & $0.03(0.1)$ & $12.9(9.2)$ & $0.07(0.2)$ & $68.8 * * *$ \\
\hline \multicolumn{5}{|c|}{ Years in General Nursing (mean, s.d.) } & $\mathrm{F}(1,204)$ \\
\hline & $7.7(10.5)$ & $23.1(9.0)$ & $6.1(9.3)$ & $15.6(12.5)$ & $27.3 * * *$ \\
\hline
\end{tabular}


labelled as Stigma towards Psychiatric Patients (STPP). For the present study, items included "mental patients should be kept behind locked doors" and "a foolish woman would marry a man with mental illness", among others. Responses were on a 5-pointscale: “1” for "disagree”, “2” for "tend to disagree”, “3” for “ neither”, “4” for "tend to agree" and "5" for "agree".

\subsection{Procedure}

Recruitment of participants was achieved through a snowballing technique. After ethics approval for the study was granted by the University of Melbourne Human Research Ethics Committee (HERC No. 020030), an initial pool of general and psychiatric nurses $(n=20)$ of Chinese-Australian and Anglo-Australian backgrounds working in various institutions were identified and asked to participate in the study. Nurses in the initial pool known to the first author, $\mathrm{Ku}$, were asked to talk with potential participants and ask permission for the first author to approach them to introduce the study formally. Those nurses who expressed an interest in participating were asked to meet with the first author for the purpose of further explanation of the nature, purpose and procedure of the study. All participants signed a written consent form to anonymous participation. Data were collected in the latter part of 2002 and early 2003.

\subsection{Response Rate}

Three hundred and forty (347) surveys were disturbed. Two hundred and eight nurses out of 331 relevant participants returned the survey giving a $63 \%$ response rate $(208 / 331 \times 100)$.

\subsection{Statistical Analysis}

Principal component analyses (PCA) were used to identify common dimensions underlying the variation of the item scores of the STPP. Cronbach's alpha coefficient was calculated to estimate the internal reliability of the derived STPP.A Two-way analysis of variance was used to examine the discriminant validity of the STPP. All analyses were conducted using the Statistical Package for the Social Sciences (SPSS Version 12).

\section{Results}

\subsection{Dimensions of the CPP}

A PCA analysis was conducted on the social distancing and alienation items. Table 2 shows the component matrix as only one factor was extracted according to the Kaiser criterion. This one dimension accounted for $43.5 \%$ of the variance in the items by dropping one item with low loading (that is, "mentally ill people are no more dangerous than others"). All the other nine items accounted for the high reliability (0.81, Table 2 ) relating to opinions expressed towards psychiatric patients, such as "fear of mental patients living in neighbourhood", "cannot trust psychiatric patients as baby sitters", and "foolish for women to marry mental patients". This measure, as intended, captures the construct of social distancing and alienation of those with a mental illness.

Table 2. Component matrix for the STPP scale.

\begin{tabular}{llc}
\hline Item & Component 1 & Communalities \\
\hline Q2 fear they live in neighbourhood & .84 & .70 \\
Q9 patients are difficult to like & .77 & .60 \\
Q5 don't give high responsible job & .76 & .57 \\
Q10 can't trust them as baby sitter & 74 & .55 \\
Q8 dangerous to forget their madness & .68 & .46 \\
Q7 should be immediately hospitalised & .68 & .46 \\
Q6 foolish women marry mental patients & .64 & .40 \\
Q1 keep behind locked doors & .57 & .33 \\
Q4 should be isolated from community & .51 & .26 \\
\hline Percent variance & & Total variance \\
& & $=43.5$ \\
\hline Cronbach's alpha & .81 & \\
\hline
\end{tabular}




\subsubsection{Discriminant Validity}

Two-way analysis of variance examined differences in scores between nurse groups and ethnic groups as before. The main effect of nurse type was significant $(\mathrm{F}(1,203)=22.84, p<0.001)$ as was the main effect of ethnicity $(\mathrm{F}(1,203)=36.68, p<0.001)$. Examination of the means indicated that Chinese nurses (mean $=2.38$, s.d. $=0.81)$ reported higher stigma than Anglo nurses (mean $=1.81$, s.d. $=0.64)$ and general nurses $($ mean $=2.34$, s.d. $=0.83$ ) higher than psychiatric nurses (mean $=1.87$, s.d. $=0.67$ ). Additionally, the interaction effect was also significant $(\mathrm{F}(1,203)=5.35, p<0.05)$ indicating that stigma differed across ethnic groups and nurse types. Chinese general nurses (mean $=2.80$, s.d. $=0.78$ ) scored highest on this scale than the other three groups of nurses: Chinese psychiatric nurses (mean $=2.08$, s.d. $=0.71$ ), Anglo general nurses $($ mean $=1.96$, s.d. $=0.66$ ), and Anglo psychiatric nurses (mean $=1.73$, s.d. $=0.61$ ), as evident in Figure 1.

It is of interest to examine directly the proportion of nurses that may have social distancing attitudes towards the mentally ill. This was done of the level items from the STPP scale to also appreciate the variation of endorsement across the items. Results are shown in Table 3 representing percentages of the sample disagreeing or agreeing with the 10 statements (including the one omitted from the scale) of social distancing specifically. The original responses of "Disagree", "Tend to disagree" were coded as "Disagree", and "Tend to agree" and "Agree" were coded as "Agree". Since the last item is reverse scored the sum of "Tend to disagree" and "Disagree" is represented in the "Agree" category. Of most interest in Table $\mathbf{3}$ is that at least one in five nurses endorsed agreement with half of the items. Table 4 shows results broken down by ethnicity and nurse type. Across

\section{Expressed Stigma by Nurses}

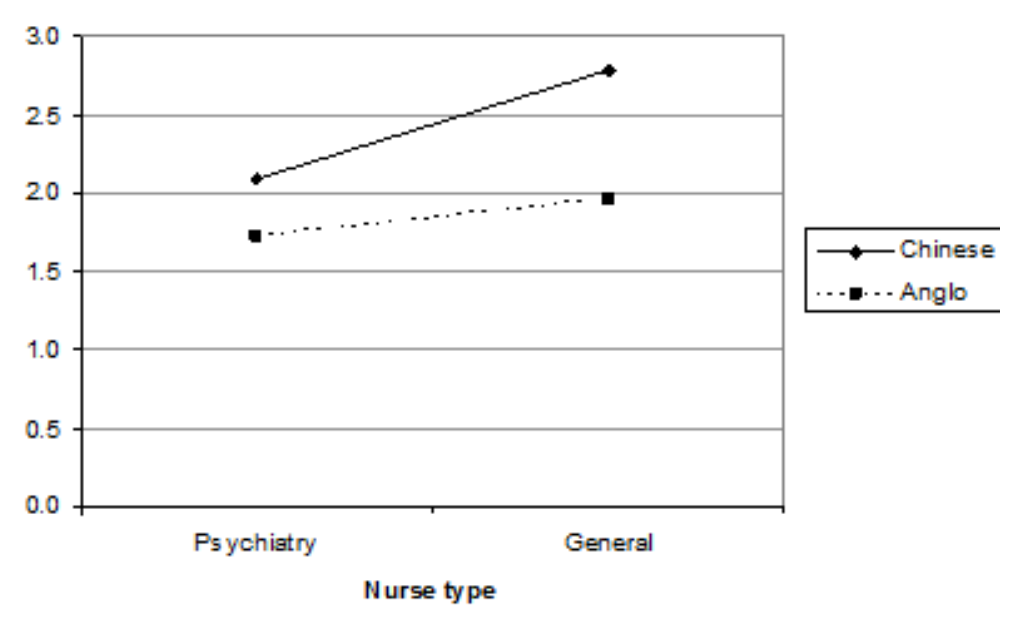

Figure 1. Social distancing by nurses.

Table 3. Stigma scale for measuring social distancing.

\begin{tabular}{|c|c|c|}
\hline Item & \multicolumn{2}{|r|}{$\begin{array}{l}\text { Agree } \\
\text { Fre }\end{array}$} \\
\hline Should be isolated from community & $189(90.0)$ & $6(2.9)$ \\
\hline * Keep behind locked doors & $187(89.9)$ & $9(4.3)$ \\
\hline Patients are difficult to like & $167(80.3)$ & $20(9.6)$ \\
\hline Should be immediately hospitalised & $167(80.3)$ & $23(11.1)$ \\
\hline Fear they live in neighbourhood & $150(72.1)$ & $25(12)$ \\
\hline Dangerous to forget their madness & $133(63.9)$ & $41(19.7)$ \\
\hline Foolish women marry mental patients & $113(54.3)$ & $47(22.6)$ \\
\hline Don't give high responsibility job & $136(65.4)$ & $52(25)$ \\
\hline No more dangerous than others ${ }^{3}$ & $113(54.3)$ & $63(44.7)$ \\
\hline Can't trust them as baby sitters & $84(40.4)$ & $72(34.6)$ \\
\hline
\end{tabular}

${ }^{1}$ Recode "Disagree” and “Tend to disagree” to "Disagree” (0); ${ }^{2}$ Recorde "Tend to agree” and “Agree” to "Agree” (1); ${ }^{3}$ Recorde scored item. ${ }^{*}$ Omitted item from the scale. 
Table 4. Percent agreement on items from the STPP scale $(\mathrm{N}=208)$.

\begin{tabular}{|c|c|c|c|c|c|c|c|c|c|}
\hline & \multicolumn{5}{|c|}{$\begin{array}{c}\text { Effect of Ethnicity \& Nurse Type } \\
\text { Agree }(\%)\end{array}$} & \multicolumn{2}{|c|}{$\begin{array}{c}\text { Effect of Ethnicity } \\
\text { Agree }(\%)\end{array}$} & \multicolumn{2}{|c|}{$\begin{array}{c}\text { Effect of Nurse Type } \\
\text { Agree (\%) }\end{array}$} \\
\hline & $\begin{array}{l}\text { Chinese } \\
\text { Psychiatry } \\
(n=49)\end{array}$ & $\begin{array}{l}\text { Chinese } \\
\text { General } \\
(n=35)\end{array}$ & $\begin{array}{l}\text { Anglo } \\
\text { Psychiatry } \\
\text { (n=83) }\end{array}$ & $\begin{array}{l}\text { Angle } \\
\text { General } \\
(n=41)\end{array}$ & $\chi^{2}$ & $\begin{array}{l}\text { Chinese } \\
(n=84)\end{array}$ & $\begin{array}{c}\text { Anglo } \\
(n=124)\end{array}$ & $\begin{array}{l}\text { Psychiatry } \\
(n=132)\end{array}$ & $\begin{array}{l}\text { General } \\
(n=76)\end{array}$ \\
\hline Should be isolated from community & 2.0 & 12.9 & 1.3 & 0.0 & $12.2^{* *}$ & 6.3 & 0.9 & 1.6 & 5.9 \\
\hline Keep behind locked doors & 6.3 & 12.1 & 2.6 & 0.0 & $7.1 \mathrm{~ns}$ & 8.6 & 1.7 & 4.0 & 5.6 \\
\hline Patients are difficult to like & 8.9 & 36.7 & 1.3 & 11.4 & $28.5^{* * *}$ & 20.0 & 4.5 & 4.1 & 23.1 \\
\hline Should be immediately hospitalized & 19.6 & 31.0 & 3.9 & 5.3 & $18.7^{* * *}$ & 24.0 & 4.3 & 9.8 & 16.4 \\
\hline Fear they live in neighbourhood & 9.3 & 48.4 & 2.9 & 12.9 & $37.8^{* * *}$ & 25.7 & 5.9 & 5.3 & 30.6 \\
\hline Dangerous to forget their madness & 22.2 & 50.0 & 7.5 & 34.4 & $23.4^{* * *}$ & 33.3 & 16.2 & 11.5 & 34.2 \\
\hline Foolish women marry mental patients & 42.9 & 51.9 & 22.2 & 11.4 & $16.6^{* *}$ & 46.8 & 18.4 & 22.6 & 29.0 \\
\hline Don't give high responsibility job & 38.1 & 64.7 & 10.7 & 16.2 & $38.9 * * *$ & 50.0 & 12.5 & 20.5 & 39.4 \\
\hline Can't trust as baby sitter & 52.4 & 92.6 & 23.3 & 40.7 & $36.9^{* * *}$ & 68.1 & 28.7 & 35.3 & 66.7 \\
\hline No more dangerous than others ${ }^{1}$ & 25.5 & 53.6 & 31.9 & 50.0 & $9.1^{*}$ & 36.0 & 37.5 & 29.4 & 51.7 \\
\hline
\end{tabular}

items it is evident that more of the Chinese and the general nurses endorsed social distancing and alienation towards the mentally ill than the Anglo group and psychiatric nurses, respectively.

\subsubsection{Association between Stigma (STPP) and Other Factors}

Correlations between Social Distancing and other measures (background demographics) are summarized in Table 5. These are direct correlations without taking account possible confounding between variables. Social distance and alienation of the mentally ill (STPP) is positively correlated with, particularly, age, years in general and mental health nursing.

\subsubsection{Relationship between Group Membership and STPP Scores}

A Two-way analysis of covariance was used to examine differences in scores between nurse types and ethnic groups while accounting for the contribution of background, and contact factors, respectively. As shown in Model 1 (Table 6) when background demographics and work experience were entered as covariates, the main effects were statistically significant according to nurse type $(\mathrm{F}(1,188)=6.50, p<0.05)$ and ethnicity $(\mathrm{F}(1,188)=$ 22.72, $p<0.001$ ). Inspection of the means indicated that Chinese nurses (mean $=2.37$, s.d. $=0.79$ ) reported higher stigma than Anglo nurses $($ mean $=1.80$, s.d. $=0.64)$. Chinese general nurses $($ mean $=2.74$, s.d. $=0.76)$ reported higher stigma than Chinese psychiatric nurses (mean $=2.11$, s.d. $=0.72$ ). Anglo general nurses (mean = 1.95 , s.d. $=0.66)$ also reported higher stigma than Anglo psychiatric nurses $($ mean $=1.72$, s.d. $=0.61)$. The interaction term was not significant $(\mathrm{F}(1,188)=2.87, p>0.05)$.Compared with the Simple Model (where background factors were not in the model) the effect of nurse type was much reduced but not eliminated by the inclusion of background covariates. Similarly, the effect of ethnicity was reduced but not eliminated by the inclusion of these background factors. Each individual covariate such as age $(\mathrm{F}(1,188)=1.36, p>0.05)$, and (sex, years in mental nursing, and years in general nursing $(F(1,188)=<1)$ ) had no simple effect.

In Model 2, contact type scores were added to background demographics as covariates. The main effect of nurse type was now not significant $(\mathrm{F}(1,184)=<1)$. However, the main effect of ethnicity remained as significant $(\mathrm{F}(1,184)=14.46, p<0.001)$. This is an indication that background demographics but particularly contact factors are important correlates of stigma and account entirely for the effect of nurse type. Examination of the means adjusted for the effects of covariates suggested that Chinese nurses (mean $=2.37$, s.d. $=0.79$ ) again reported higher stigma than Anglo nurses (mean $=1.80$, s.d. $=0.64$ ). The interaction effect was not significant $(\mathrm{F}(1,184)=2.13, p>0.05)$. Each demographic and contact factor had no simple effect: age $(\mathrm{F}(1,184)=1.12, p>$ 0.05 , contact via work $(\mathrm{F}(1,184)=1.69, p>0.05)$, patients helping nurse $(\mathrm{F}(1,184)=2.97, p>0.05)$, and (sex, years in mental health nursing, years in general nursing, relatives with mental illness, and socialising with person with mental illness $(\mathrm{F}(1,184)=<1)$ ).

The nurse type effect on social distancing is most probably due to the combined influence of background demographics, work experience, and contact levels with mental illness. To some extent these factors also medi- 
Table 5. Pearson correlations between general stigma and background measures.

\begin{tabular}{ll}
\hline & $\begin{array}{c}\text { Social Distancing } \\
\text { (STPP Score) }\end{array}$ \\
\hline Background Factors & $.18^{*}$ \\
AGE & $.13 \mathrm{~ns}$ \\
SEX & $-.16^{*}$ \\
YR_MH_NU & $.26^{* * *}$ \\
YR_GE_NU & $-.39^{* * *}$ \\
\hline Contact Factors & $-.30^{* * *}$ \\
CWS & $-.14^{*}$ \\
PHN & $-.32^{* * *}$ \\
RMI & \\
ESP & ${ }^{*}<<0.05,{ }^{* * *} p<0.001 ;$ ns, not significant. YR_MH_NU = Years in Mental Health Nursing; YR_GE_NU = Years \\
in General Nursing; CWS = Contact Through Work Situation; PHN = Patient Helps Nurse; RMI = Relative with \\
Mental Illness; ESP = External Socialisation with Patient.
\end{tabular}

Table 6. Relationship between background variables and STPP scores.

\begin{tabular}{lccl} 
& \multicolumn{3}{c}{ F Values } \\
& $\begin{array}{l}\text { Simple } \\
\text { Model }\end{array}$ & $\begin{array}{c}\text { Model } 1 \\
\text { with background } \\
\text { factors } 1\end{array}$ & $\begin{array}{l}\text { Model } 2 \\
\text { with background } \\
\text { and contact factors }{ }^{2}\end{array}$ \\
\hline Nurse type & & $6.50^{*}$ & $0.12 \mathrm{~ns}$ \\
Ethnic group & $22.84^{* * *}$ & $22.72^{* * *}$ & $14.46^{* * *}$ \\
Interaction & $36.68^{* * *}$ & $2.87 \mathrm{~ns}$ & $2.13 \mathrm{~ns}$ \\
\hline
\end{tabular}

${ }^{*} p<0.05,{ }^{* *} p<0.01,{ }^{* * *} p<0.001$; ns, not significant. ${ }^{1}$ covariates = age, sex, years of mental nursing experience, years of general nursing experience; ${ }^{2}$ covariates = age, sex, years of mental nursing experience, years of general nursing experience, and contact levels [(CPP Scale = CWS (via work), PHN (patients helping nurse), RMI (relatives with mental illness), ESP (socializing with person with mental illness)).

ate the ethnicity effect on social distancing. An additional non-mediated effect on social distancing, or some unmeasured factor is also strongly related to ethnicity is related to social distancing.

\section{Discussion}

Since stigma is so socially pervasive, crossing national, social and cultural boundaries, it is legitimate to ask what its effects on those who have a role in the treatment and support of the mentally ill are. Are such professionals less prone to stigmatizing attitudes and if not do such attitudes influence their approach to patient care? Such questions, particularly the last one, have been rarely raised in the international literature and therefore constitute a key focus of this research. Registered nurses were chosen in this study as they were the largest workforce in health delivery system in comparison with doctors, psychologists, social workers and occupational therapists. Nurses' attitudes towards mental illness have considerable influence in relation to patients' response towards their treatment adherence, rehabilitation and recovery process.

In the present study, stigma was measured by social distancing and alienation of the mentally ill (STPP Scale). Nurses seemed to highly endorse STPP and DISL items similar to what could be expected for the general public. For example, it was shown that for several of the STPP items, as many as 20 and 40 percent of nurses endorsed these. Particularly items that suggested the theme of dangerousness (e.g., "not allowing a person with mental illness to baby sit”) seemed to be highly endorsed. These findings are similar to the results of Caldwell and Jorm (2001) [8] and Lauber et al. (2006) [9] although those studies were done with other professional groups. As indicated in the previous work, Lauber et al. (2006) [9] found that psychiatrists endorsed more stigmatizing attitudes than other professionals (psychologists, nurses) including higher negative stereotype endorsement. It seems that professionals in a role to provide care to people with mental illness are no less likely to carry stigmatizing attitudes towards those with a mental illness.

It is shown clearly and presently that endorsement of social distancing was lower among psychiatric nurses. This might be expected, to some extent, because psychiatric nurses had more experience (by definition of the groups in this study) of people with mental illness and therefore they may feel safe in their knowledge and skills for interacting with the mentally ill, because they have a personal interest in caring for the mentally ill, as shown by their choice of specialty, or, because they have experienced people with mental illness in a wider variety of 
settings and roles (as measured by the Contact Scale (CPP, Contact with psychiatric Patient).Further, the nurse type effect was reduced by controlling for differences based on background factors (including amount of mental health nursing experience) and contact level. Indeed, once accounting for these factors, there were no differences in general stigma between two nurse type groups. This implies that contact, experience and choice of specialty may be significant determinants of general stigma towards people with a mental illness amongst nurses. There have not been any other comparative studies between general and psychiatric nurses to be able to suggest that these findings generalize to other settings. The few studies of other disciplines show mixed results (Jorm et al., 1999 [10]; Kua et al., 2000 [11]; Caldwell \& Jorm, 2001[8]).

In Caldwell and Jorm's (2001) [8] study, for example, psychiatric nurses were more optimistic about outcomes for the mentally ill than other professionals. However, these professional groups also included psychiatrists and clinical psychologists who may be similar to the psychiatric nurses in choosing mental health as their specialty. Kua et al. (2000) [11] on the other hand compared general medical practitioners and psychiatrists. Findings suggested more optimism amongst psychiatrists who endorsed better outcomes for people with mental illness, consistent in principle with present findings for nurses. However, clearly, these previous studies measured constructs that could be related to stigmatizing attitudes but did not directly measure such attitudes. On the other hand, studies of mental health training of generalist health care professionals (Chinnayya et al., 1990) [12], students nurses (Evagelou et al., 2005[13]; Madianos et al., 2005) [14] and medical students have shown some reductions in negative attitudes towards mentally ill individuals. Such results are consistent with present findings, but are more specifically indicative of the effects of training exposure and short term experience on attitudes rather than effects due to choice of specialty. However, training effects have been inconsistent and are probably due to specific factors (Reed \& Fitzgerald, 2005) [15], and may not last (Sivakumar et al., 1986) [16] and Baxter et al. (2001) [17].

The results from comparing mental health professionals and public attitudes in Lauber's et al. (2006) [9] work revealed that psychiatrists were said to hold as negative an attitude towards the mentally ill as was held by the general public. This is not consistent with the present findings that argue that experience, knowledge and contact should be related to less stigmatizing attitudes. 0 .

Social distancing in current study varied according to cultural group membership of the nurses. Chinese nurses, particularly general nurses, were shown to endorse more highly social distancing towards those with mental illness and contact was placed as a mediator for this attitude (Ku, Ha \& Siriwan, 2015) [18]. Results suggest a relationship between culture and stigma, specifically among Chinese nurses but do not clarify why, such a relationship exists (Ku \& Ha, 2015) [19].None of the previous studies quoted earlier effectively show cultural differences in stigma as they mainly dealt with within-culture depiction of social distancing towards people with a mental illness. This had prompted the first author, Ku, to design the Attitudes towards Depression and Schizophrenia Scale (ADSS) to measure Chinese Australian Community Attitudes towards Mental Illness (Ku\& Ha, 2015) [20].

\section{Conclusion}

The findings in this study suggest that the STPP is reliable in measuring nurses' attitudes towards mental illness due to its high internal consistency. Results suggest that Chinese group membership influence social distancing based on threat possibilities.

\section{Acknowledgements}

The authors would like to thank Victoria University Institute of Technology and XianJiaotong-Liverpool University for supporting and sponsoring the presentation of this paper.

\section{Authors' Contributions}

The first author carried out the research and prepared the first draft and the second author verified the statistical results and edited the paper. Both authors have approved the final version of the manuscript.

\section{References}

[1] New Webster’s Dictionary and Thesaurus and Medical Dictionary (1992). Ottenheimer Publishers, Inc., New York. 
[2] Goffman, E. (1970) Stigma: Notes on the Management of Spoiled Identity. Penguin Books Ltd., Harmondsworth, UK.

[3] Angermeyer, M.C. and Schulze, B. (2001) Reinforcing Stereotypes: How the Focus on Forensic Cases in News Reporting May Influence Public Attitudes towards the Mentally Ill. International Journal of Law and Psychiatry, 24, 469-486. http://dx.doi.org/10.1016/S0160-2527(01)00079-6

[4] Rabkin, J.G. (1974) Public Attitudes towards Mental Illness: A Review of the Literature. Schizophrenia Bulletin, 10, 9-33. http://dx.doi.org/10.1093/schbul/1.10.9

[5] Link, B.G., Mirotznik, J. and Cullen, F.T. (1991) The Effectiveness of Stigma Coping Orientations: Can Negative Consequences of Mental illness Labelling Be Avoided? Journal of Health and Social Behaviour, 32, 302-320. http://dx.doi.org/10.2307/2136810

[6] Lin, T. (1983) Psychiatry and Chinese Culture. The Western Journal of Medicine, 139, 862-867.

[7] Cohen, J.S. and Struening, E.L. (1962) Opinions about Mental Illness in the Personnel of Two Large Mental Hospitals. Journal of Abnormal and Social Psychology, 64, 349-360. http://dx.doi.org/10.1037/h0045526

[8] Caldwell, T.M. and Jorm, A.F. (2001) Mental Health Nurses' Beliefs about Likely Outcomes for People with Schizophrenia or Depression: A Comparison with the Public and Other Health Care Professionals. Australian and New Zealand Journal of Mental Health Nursing, 10, 42-54. http://dx.doi.org/10.1046/j.1440-0979.2001.00190.x

[9] Lauber, C., Nordt, C., Braunschweig, C. and Rossler, W. (2006) Do Mental Health Professionals Stigmatize Their Patients? Acta Psychiatrica Scandinavica, 113, 51-59. http://dx.doi.org/10.1111/j.1600-0447.2005.00718.x

[10] Jorm, A.F., Korten, A.E., Jacomb, P.A., Christensen, H. and Henderson, S. (1999) Attitudes towards People with a Mental Disorder: A Survey of the Australian Public and Health Professionals. Australian and New Zealand Journal of Psychiatry, 33, 77-83. http://dx.doi.org/10.1046/j.1440-1614.1999.00513.x

[11] Kua, J.H.K., Parker, G., Lee, C. and Jorm, A.F. (2000) Beliefs about Outcomes for Mental Disorders: A Comparative Study of Primary Health Practitioners and Psychiatrists in Singapore. Singapore Medical Journal, 41, 542-547.

[12] Chinnayya, H.P., Chandrashekar, C.R., Moily, S. Puttamma , Raghuram, A., Subramanya, K.R., Shanmugham, V. and Udaykumar, G.S. (1990) Training Primary Care Health Workers in Mental Health Care: Evaluation of Attitudes towards Mental Illness before and after Training. International Journal of Social Psychiatry, 36, 300-307. http://dx.doi.org/10.1177/002076409003600408

[13] Evagelou, H., Adali, E., Koukia, E., Katostaras, F., Priami, M. and Toulia, G. (2005) The Influence of Education of Nursing Students on the Formation of Attitudes toward Psychiatric Illness. ICUS and Nursing Web Journal, Issue 23, $1-8$.

[14] Madianos, M., Priami, M., Aleviscopoulous, G., Koukia, E. and Rogakou, E. (2005) Nursing Students’ Attitudes Change towards Mental Illness and Psychiatric Case Recognition after a Clerkship in Psychiatry. Issues in Mental Health Nursing, 26, 169-183. http://dx.doi.org/10.1080/01612840590901635

[15] Reed, F. and Fitzgerald, L. (2005) The Mixed Attitudes of Nurse's to Caring for People with Mental Illness in a Rural General Hospital. International Journal of Mental Health Nursing, 14, 249-257. http://dx.doi.org/10.1111/j.1440-0979.2005.00389.x

[16] Sivakumar, K., Wilkinson, G., Toone, B. and Greer, S. (1986) Attitudes to Psychiatry in Doctors at the End of Their First Post-Graduate Year: Two-Year Follow-Up of a Cohort of Medical Students. Psychological Medicine, 16, 457-460. http://dx.doi.org/10.1017/S0033291700009296

[17] Baxter, H., Singh, S.P., Standen, P. and Duggan, C. (2001) The Attitudes of “Tomorrow’s Doctors” towards Mental Illness and Psychiatry: Changes during the Final Undergraduate Year. Medical Education, 35, 381-383. http://dx.doi.org/10.1046/j.1365-2923.2001.00902.x

[18] Ku, T.K., Ha, M. and Siriwan, U. (2015) Professional Attitudes towards Mental Illness: Testing the Contact Hypothesis among Nurses of Ethnic Anglo and Chinese Australian Backgrounds. Journal of Social Sciences, 3, 130-138. http://dx.doi.org/10.4236/jss.2015.37022

[19] Ku, T.K. and Ha, M. (2015) Culture and Stigma of Mental Illness: Path Analysis Conducted with AMOSin Transcultural Psychiatry in Australia. Journal of Biosciences and Medicines, 3, 15-22. http://dx.doi.org/10.4236/jbm.2015.36003

[20] Ku, T.K. and Ha, M. (2015) Development of the Attitudes towards Depression and Schizophrenia Scale (ADSS). Studies in Social Sciences and Humanities, 2, 12-20. 